\title{
Caracterização epidemiológica das vítimas de acidentes motociclísticos atendidas no estado do Piauí
}

\author{
Epidemiological characterization of motorcycle accident victims treated in the state of Piauí
}

Caracterización epidemiológica de las víctimas de accidentes motociclísticos atendidas en el estado de Piauí

\begin{abstract}
Vanessa Oliveira do Monte ${ }^{1 *}$, Maria Amélia de Oliveira Costa ${ }^{1}$, Matheus Henrique da Silva Lemos ${ }^{1}$, Taciany Alves Batista Lemos ${ }^{1}$, Camilla Lemos Morais ${ }^{1}$, Thaysa Batista Vieira de Rezende ${ }^{1}$, Maysa Águida Silva Lima ${ }^{1}$, Antonio de Lima de Sousa Júnior ${ }^{1}$, Veridiana Mota Veras ${ }^{1}$, Liliana Negreiros Silva Chaves ${ }^{1}$, Bruna Furtado Sena de Queiroz ${ }^{1}$, Isaias Silva Costa ${ }^{1}$, Jayris Lopes Vieira1.
\end{abstract}

\section{RESUMO}

Objetivo: Caracterizar o perfil epidemiológico das vítimas de acidentes motociclísticos atendidos na rede hospitalar no estado do Piauí. Métodos: Estudo descritivo, retrospectivo de abordagem quantitativa, com dados secundários do Sistema de Internação Hospitalar (SIH) do Departamento de Informática do Sistema Único de Saúde (DATASUS). A coleta de dados foi realizada no período de setembro a outubro de 2018 e foram analisadas as seguintes variáveis: faixa etária, sexo, cor/raça, número de óbitos, mês de ocorrência, mecanismo causador, estabelecimento de ocorrência e a média de permanência. A organização e análise dos dados ocorreu no mês de novembro de 2018 através do programa TABNET. Resultados: Foram registrados 5.680 acidentes com motociclistas no ano de 2017, sendo que a maioria está entre a faixa de 20 a 39 anos, de cor parda e do sexo masculino. Houve a predominância no mecanismo causador de acidente de trânsito não especificado e o estabelecimento que apresentou o maior número de vítimas foi o Hospital de Referência do município de Teresina, no mês de janeiro. Conclusão: $O$ estudo demostrou os elevados índices de acidentes com motociclistas no estado do Piauí, evidenciando a necessidade da implantação de políticas públicas voltadas para a conscientização e educação no trânsito.

Palavras-Chave: Motocicletas, Causas externas, Epidemiologia.

\begin{abstract}
Objective: Characterize the epidemiological profile of the victim's motorcycle accidents attended in the hospital network in the state of Piauí. Methods: A descriptive, retrospective study of a quantitative approach, with secondary data from the Hospital Inpatient System (SIH) of the Department of Informatics of the Unified Health System (DATASUS). Data collection was performed from September to October 2018 and the following variables were analyzed: age, sex, color / race, number of deaths, month of occurrence, causative mechanism, establishment of occurrence and mean permanence. The organization and analysis of the data occurred in the month of November 2018 through the program TABNET. Results: There were 5,680 accidents with motorcyclists registered in 2017, with the majority being between the ages of 20 and 39, of brown and male color. There was a predominance in the mechanism causing an unspecified traffic accident and the establishment that presented the largest number of victims was the Reference Hospital of the municipality of Teresina, in January. Conclusion: The study showed the high rates of accidents with motorcyclists in the state of Piauí, evidencing the need to implement public policies focused on awareness and education in traffic.
\end{abstract}

Key words: Motorcycles, Accidents, Epidemiology.

${ }^{1}$ Faculdade Integral Diferencial - FACID/WYDEN. Teresina, Piauí. *E-mail: vanessamonte_02@hotmail.com

SUBMETIDO EM: 4/2019

ACEITO EM: 5/2019

PUBLICADO EM: 7/2019

REAS/EJCH | Vol. Sup. 25 | e738 | DOI: https://doi.org/10.25248/reas.e738.2019 Página 1 de 8 


\section{RESUMEN}

Objetivo: Caracterizar el perfil epidemiológico de las víctimas de accidentes motociclísticos atendidos en la red hospitalaria en el estado de Piauí. Métodos: Estudio descriptivo, retrospectivo de abordaje cuantitativo, con datos secundarios del Sistema de Internación Hospitalaria $(\mathrm{SIH})$ del Departamento de Informática del Sistema Único de Salud (DATASUS). La recopilación de datos fue realizada en el período de septiembre a octubre de 2018 y se analizaron las siguientes variables: grupo de edad, sexo, color / raza, número de muertes, mes de ocurrencia, mecanismo causante, establecimiento de ocurrencia y el promedio de permanencia. La organización y análisis de los datos ocurrió en el mes de noviembre de 2018 a través del programa TABNET. Resultados: Se registraron 5.680 accidentes con motociclistas en el año 2017, siendo que la mayoría está entre el rango de 20 a 39 años, de color parda y del sexo masculino. Se ha predominado en el mecanismo causante de accidentes de tránsito no especificado y el establecimiento que presentó el mayor número de víctimas fue el Hospital de Referencia del municipio de Teresina, en el mes de enero. Conclusión: El estudio demuestra los altos índices de accidentes con motociclistas en el estado de Piauí, evidenciando la necesidad de la implantación de políticas públicas dirigidas a la concientización y educación en el tránsito.

Palabras clave: Motocicletas, Causas externas, Epidemiología.

\section{INTRODUÇÃO}

Os acidentes de trânsito são um grave problema mundial, pois causam sérios impactos sociais, psicológicos, econômicos, previdenciários, ambiental e no âmbito do setor saúde, devido ao impacto na morbidade e na mortalidade, principalmente da população jovem e do sexo masculino. Segundo a Organização Mundial da Saúde (OMS), em 2013, as mortes e as lesões no trânsito foram responsáveis por um custo mundial de $3 \%$ do Produto Interno Bruto (PIB), enquanto nos países com renda baixa e média, estimou-se que esse custo foi em torno de $5 \%$ do PIB (OMS, 2015).

O cenário brasileiro mostra que os acidentes de transporte terrestre correspondem à segunda causa de morte entre as causas externas, prevalecendo entre jovens e adultos de 15 a 39 anos (BRASIL, 2017). Segundo o Ministério da Saúde, no ano de 2013, foram registrados 45.099 óbitos e 228.400 internações por ATT que geraram um custo de $R \$ 303,5$ milhões para o Sistema Único de Saúde (SUS) (BRASIL, 2015).

É considerado um problema multifatorial que é resultante da combinação de fatores relacionados às vias, ao ambiente, aos veículos, aos usuários e ao modo como eles interagem. Destacando-se o aumento da frota de veículos circulantes, comportamentos inadequados no trânsito, a fiscalização é insuficiente e descontínua. Esses fatores são responsáveis por boa parte das mortes precoces e a principal causa de traumatismos, sequelas e incapacidades no Brasil (BRASIL, 2017).

O uso da motocicleta como meio de transporte tem-se modificado, tanto por fatores culturais como por vantagens econômicas tais como: custo mais baixo tanto do veículo como para manutenção do mesmo, além de facilitar o deslocamento principalmente em rodovias congestionadas. As motocicletas são consideradas uma eficiente ferramenta de transporte e o trabalho vem invadindo o espaço urbano, tornando o trânsito de grandes cidades congestionadas (ANDRADE ML, 2009; CORGOZINHO MM et al., 2018).

Entre os acidentes de trânsito no Brasil, a incidência vem aumentando principalmente aos relacionados com motocicleta, visto que a população opta por ser um meio de transporte mais rápido e de baixo custo. Os condutores de motocicletas são considerados prioritários nos programas de prevenção em que apresentam riscos, sete vezes maior, de morte, quatro vezes maior, de lesão corporal e duas vezes maior, de atropelar um pedestre, comparando aos meios automobilísticos (MELO LMF et al., 2015).

Vale ressaltar que $80 \%$ dos acidentes com motocicletas ocasionam lesões, sendo as extremidades a região mais acometida, visto que é a região mais desprotegida. Os motociclistas estão mais vulneráveis, principalmente quando em colisão com veículos maiores (SILVA PHNV et al., 2011; RODRIGUES CL et al., 2014). 
Segundo o Relatório Global sobre o Estado da Segurança Viária 2015, da Organização Mundial da Saúde (OMS), foram registradas no país 42.291 mortes no trânsito (82\% homens e 18\% mulheres) em 2013. O maior número de mortes (28\%) acontece com usuários de veículos com 2 ou 3 rodas, como é o caso das motocicletas. Pedestres também estão na lista dos mais atingidos no trânsito: $20 \%$ dos óbitos. Além disso, 18\% dos óbitos acontecem com passageiros de veículos de quatro rodas e 3\% com ciclistas (OMS, 2015).

Diante do exposto, este estudo teve como objetivo caracterizar o perfil epidemiológico das vítimas de acidentes moto ciclísticos atendidos na rede hospitalar no estado do Piauí.

\section{MÉTODOS}

Tratou-se de um estudo descritivo, retrospectivo de abordagem quantitativa, com dados secundários do Sistema de Internação Hospitalar $(\mathrm{SIH})$ do Departamento de Informática do Sistema Único de Saúde (DATASUS).

O estudo abrangeu todos os casos de vítimas de acidentes moto ciclísticos traumatizados atendidos na rede hospitalar e registrados no SIH-DATASUS no Estado do Piauí, no ano de 2017. Foram incluídas as informações estatísticas referente as internações das vítimas de acidentes moto ciclísticos traumatizados atendidos em serviços de saúde no ano de 2017. Foram excluídos os dados de vítimas de acidentes que não foram ocasionados por moto atendidos em unidades de trauma antes do ano de 2017 ou que não foram atendidos no Estado do Piaui.

A coleta de dados foi realizada no período de setembro a outubro de 2018. Foram analisadas as seguintes variáveis: faixa etária, sexo, cor/raça, taxa de mortalidade, número de óbitos, mês de ocorrência, mecanismo causador, estabelecimento de ocorrência e a média de permanência.

A organização e análise dos dados ocorreu no mês de novembro de 2018, através do programa TABNET. Em seguida os resultados foram exportados para o software Microsoft Excel $\AA$, sendo realizado análise estatística descritiva através números absolutos e percentuais, sendo organizados em tabelas e quadros. Tais dados foram confrontados com a literatura cientifica existente sobre a temática.

O presente estudo foi realizado através de dados públicos, retirado do banco de dados SIH-DATASUS, dispensando a necessidade de enviar o projeto à Plataforma Brasil e ao Comitê de Ética e Pesquisa (CEP). Apesar disso, todas as resoluções enumeradas pela resolução 466/12 do Conselho Nacional em Saúde (CNS), serão atendidas, no que se refere às pesquisas envolvendo seres humanos.

\section{RESULTADOS E DISCUSSÃO}

Foram notificados 5.680 acidentes motociclísticos atendidos no estado do Piauí, no período de 2017. No Brasil a prevalência de acidentes de moto vem aumentando significativamente, elevando assim a morbimortalidade, gerando assim grandes custos para o Sistema Único de Saúde e tornando-se um problema de saúde pública (OLIVEIRA NLB, SOUSA RMC, 2012).

A Tabela 1 apresenta a frequência das variáveis epidemiológicas de vítimas de acidente de moto traumatizados atendidos no Piaú, na qual o sexo masculino foi o predominante com $81,84 \%$ (4643), a faixa etária de 20 a 39 anos foi a que apresentou o maior número de casos com 55,33\% (3143) e cor/raça parda foi a prevalente com $30,88 \%$ (1754), apesar de que há uma grande porcentagem de $67,22 \%$ sem informações relativas a essa variável, mostrando que existe uma subnotificação no preenchimento. Em relação ao sexo, foi possível observar que o sexo masculino $(81,74 \%)$ se mostrou preponderante em relação ao sexo feminino $(18,26 \%)$, dados que se assemelham ao estudo realizado no Piauí por Soares et al. (2015), onde mostrou que $88,7 \%$ dos acidentados era do sexo masculino. Estudo de Mascarenhas e Barros (2011) também realizado a nível nacional, mostrou a predominância no sexo masculino $(70,4 \%)$ e na faixa etária de 20 a 39 anos (36,9\%), quando também analisaram dados do Sistema de Informações Hospitalares do Sistema Único de Saúde (SIH/SUS). 
Tabela 1 - Distribuição das vítimas de acidentes motociclísticos atendidas no estado do Piauí segundo sexo, idade e cor/raça.

\begin{tabular}{cll}
\hline Variável & $\mathbf{N}$ & $\%$ \\
\hline Sexo & & \\
Masculino & 4643 & $81,74 \%$ \\
Feminino & 1037 & $18,26 \%$ \\
\hline Idade & & \\
$0-9$ anos & 86 & $1,51 \%$ \\
$10-19$ anos & 710 & $12,50 \%$ \\
$20-39$ anos & 3143 & $55,33 \%$ \\
$40-59$ anos & 1402 & $24,68 \%$ \\
$>60$ & 339 & $5,97 \%$ \\
\hline Cor/raça & & \\
Branca & 18 & $0,32 \%$ \\
Preta & 37 & $0,65 \%$ \\
Parda & 1754 & $30,88 \%$ \\
Amarela & 52 & $0,91 \%$ \\
Indígena & 1 & $0,02 \%$ \\
Sem Informações & 3818 & $67,22 \%$ \\
\hline
\end{tabular}

Fonte: $\mathrm{SIH}(2018)$.

Uma pesquisa realizada em um hospital de referência do município de Teresina, a qual utilizou a análise de prontuários, observou-se que em relação à faixa etária, houve a predominância entre 21 a 39 anos, na qual os dados corroboram com os resultados encontrados no presente estudo (COSTA GS, 2018). Os jovens são mais vulneráveis a esse evento, devido à falta de experiência e a atribuição da condução de veículo a diversão, assim não refletindo as consequências do seu comportamento (SILVEIRA JZM, SOUZA JC, 2016).

O estudo mostra que homens jovens são os principais grupos envolvidos em acidentes de moto, por tratarse de uma população economicamente ativa no país, o que causa um grande impacto no âmbito socioeconômico, pois sequelas decorrentes do trauma podem incapacitar esse grupo, assim comprometendo a qualidade de vida e eventuais perdas de força laborativa futura (SIMONETE FS et al., 2016).

Uma possível justificativa para este achado consiste no fator cultural, visto que o homem se encontra mais exposto a situações de perigo, atribuindo também ao uso de álcool e drogas ao conduzir veículos. Achados da Pesquisa Nacional de Saúde (PNS), representativa dos adultos brasileiros, e também pesquisa do sistema de Vigilância de Fatores de Risco e Proteção para Doenças Crônicas por Inquérito Telefônico (VIGITEL), estudo representativo dos adultos residentes nas 26 Capitais Brasileiras e no Distrito Federal, demostram que ao conduzir veículos geralmente o sexo masculino tem um habito de consumir bebidas alcoólicas e conduzir veículos seja carro, motos, etc (MALTA DC, 2016).

A cor parda neste estudo mostrou um predomínio, dados que se ratificam com o estudo de Andrade e Jorge (2017), vez que segundo o Instituto Brasileiro de Geografia e Estatística (IBGE) em 2016, apontou em sua pesquisa que o predomínio da população parda prevaleceu no Nordeste.

Estudo realizado com dados do sistema de informações hospitalares do Sistema Único de Saúde (SIH/SUS), os gastos financiados pelo SUS nas internações decorrentes de acidentes com automobilísticos, no ano de 2013 no Brasil, totalizarão $\mathrm{R} \$ 231.469 .333,13$ gastos expressivos para a união, com 1.072 .557 dias de permanência e média de quase uma semana de internação por paciente, cujos dados vão de encontro com os achados desta pesquisa (ANDRADE SSCA, JORGE MHPM, 2017).

Os acidentes automobilísticos geram ônus para a união com inúmeros gastos decorrentes desde a interação até o tratamento dos pacientes (SILVEIRA JZM, SOUZA JC, 2016). Os principais fatores consistem no tempo de permanência hospitalar e os gastos decorrentes a quem necessitam de atendimento médico decorrente do trauma, podendo surgir a necessidade de cirurgias assim como de tratamentos intensivos (ANDRADE SSCA, JORGE MHPM, 2017). 
A Tabela 2 corresponde a relação entre a média de permanência e a faixa etária de vítimas de acidente de moto traumatizados atendidos no Piauí, na qual identificou-se que a média de permanência foi de 4,5 dias e as faixas etárias de 0 a 9 anos contribuiu com 6,7 dias de permanência no hospital, e o menor intervalo foi na faixa etária de 10 a 39 anos com um total de 4,3 dias. Dados de um estudo realizado no Brasil demonstrou que a média de permanência de pacientes vítimas de acidentes de trânsito foi de 6,1 dias, tempo superior ao encontrado no presente estudo (MASCARENHAS MDM, BARROS MBA, 2011).

Tabela 2 - Distribuição das vítimas de acidentes motociclísticos atendidas no estado do Piauí segundo os dias de permanência no hospital relacionado com a faixa etária.

\begin{tabular}{cc}
\hline Faixa Etária & Média de Permanência \\
\hline $0-9$ anos & 6,7 \\
$10-19$ anos & 4,3 \\
$20-39$ anos & 4,3 \\
$40-59$ anos & 4,7 \\
$>60$ anos & 4,5 \\
\hline Total & 4,5 \\
\hline
\end{tabular}

Fonte: $\mathrm{SIH}(2018)$.

A Tabela 3 demonstra a distribuição de vítimas de acidente de moto, segundo os mecanismos causadores, em que $93,59 \%$ foram acidentes de trânsito não especificado. O segundo valor prevalente foi acidentes sem colisão $2,02 \%$ e o menor causador no ano de estudo foi de colisão com outra moto e caminhão $0,03 \%$ em ambos.

Tabela 3 - Distribuição das vítimas de acidentes motociclísticos atendidas no estado do Piauí segundo o mecanismo causador.

\begin{tabular}{lll}
\hline Variável & $\mathbf{N}$ & $\%$ \\
\hline Mecanismo causador & & \\
Colisão com pedestre ou animal & 20 & $0,35 \%$ \\
Colisão com bicicleta & 5 & $0,09 \%$ \\
Colisão com outra moto & 2 & $0,03 \%$ \\
Colisão com caminhão & 2 & $0,03 \%$ \\
Colisão com ônibus & 3 & $0,05 \%$ \\
Colisão com outro veículo não motorizado & 5 & $0,09 \%$ \\
Colisão com objeto fixo & 19 & $0,33 \%$ \\
Sem colisão & 115 & $2,02 \%$ \\
Não especificado & 5316 & $93,59 \%$ \\
\hline
\end{tabular}

Fonte: SIH (2018).

Conforme o Conselho Federal de Medicina (CFM), por meio da resolução $n^{\circ} 1.638 / 2002$, aponta que em casos de emergências nas quais seja impossível a coleta da história clínica do paciente, deverá constar relato médico completo de todos os procedimentos realizados e que tenham possibilitado o diagnóstico e/ou a remoção para outra unidade. Sendo assim, entende-se que a equipe multiprofissional é responsável pelo preenchimento correto dos dados em prontuário, tendo a consciência de que as informações coletadas são importantes para garantir a continuidade da assistência, haja vista que o prontuário é um documento sigiloso e cientifico, portanto deve ter seus campos devidamente registrados (COSTA GS et al., 2018).

Simonete FS et al. (2016) demonstrou no seu estudo que as colisões representaram a maior parcela das ocorrências, e a queda de moto foi o segundo principal mecanismo causador, correspondendo a $15,4 \%$. Dados que corroboram em parte com o presente estudo, em que se considera que os acidentes sem colisões sejam justamente as quedas desses motociclistas. Uma pesquisa realizada por Costa GS et al (2018) mostrou que houve a predominância colisão moto-automóvel, o que difere do presente estudo, onde verificou-se que houve o predomínio de acidentes de trânsitos sem colisão. 
O consumo de bebidas alcoólicas é o principal fator que causa o acidente de trânsito. Elas geram dificuldades para com a tomada de decisões do condutor nas rodovias, assim como debilita suas habilidades psicomotoras, influenciando em alterações nas tarefas de condução do veículo favorecendo para que os acidentes tornem-se mais graves, consequentemente aumentando o índice de mortalidade (MALTA DC et al., 2017; LIBERATTI CLB et al. 2003).

O uso de capacete com viseiras ou óculos de proteção e vestuários de acordo com as normas e especificações aprovadas pelo o Conselho Nacional de Trânsito (CONTRAN), são equipamentos individuais que devem ser usados por condutores e passageiros de motociclistas (BRASIL, 2000). Este é considerado um equipamento eficaz nana prevenção de lesões encefálicas e de face, e na redução de sequelas (LIBERATTI CLB et al. 2003)

De acordo com o estabelecimento que mantém as vítimas de acidentes moto ciclísticos (Tabela 4), a Unidade de Referência para Urgência de alta complexidade de Teresina, teve o maior registro de acidentes, esses valores podem ser explicados porque é o referido hospital é referência no atendimento de trauma do estado do Piauí.

Tabela 4 - Distribuição das vítimas de acidentes motociclísticos atendidas no estado do Piauí segundo o estabelecimento de atendimento.

\begin{tabular}{llcc}
\hline \multicolumn{1}{c}{ Estabelecimento } & \multicolumn{1}{c}{ Município } & N & $\%$ \\
\hline Ums de Sá Bezerra & Monsenhor Hipólito & 2 & $0,03 \%$ \\
Hosp Reg Sen Dirceu Arcoverde & Água Branca & 54 & $0,95 \%$ \\
Hosp Regional Leonidas Melo & Barras & 37 & $1,00 \%$ \\
Hosp Mun Daniel Carlos de Andrade & Itaueira & 13 & $0,23 \%$ \\
Hosp de Altos Inst de Saude Jose Gil Barbosa & Altos & 6 & $0,10 \%$ \\
Ums Inhazinha Nunes & Inhuma & 6 & $0,10 \%$ \\
Hosp Reg de Bom Jesus & Bom Jesus & 75 & $1,32 \%$ \\
Hospital de Amarante & Amarante & 14 & $0,25 \%$ \\
Hospital Regional Tiberio Nunes & Floriano & 591 & $10,40 \%$ \\
Hospital Local de Guadalupe & Guadalupe & 27 & $0,48 \%$ \\
Hospital Reg Teresinha Nunes de Barros & São João do Piauí & 30 & $0,53 \%$ \\
Clinicor & Teresina & 2 & $0,03 \%$ \\
Hospital Getúlio Vargas & Teresina & 108 & $1,90 \%$ \\
Hosp Reg Sen Candido Ferraz & São Raimundo & 76 & $1,33 \%$ \\
Hosp Reg de Campo Maior & Nonato & & \\
Hosp Reg Deolindo Couto & Campo Maior & 173 & $3,05 \%$ \\
Hosp Reg Eustaquio Portela & Oeiras & 75 & $1,32 \%$ \\
Hosp Universitário da UFPI & Valença do Piauí & 1 & $0,02 \%$ \\
Unid Mista Salomão Caetano & Teresina & 0 & $0,00 \%$ \\
Hospital Regional Justino Luz & Alagoinha do Piauí & 1 & $0,02 \%$ \\
Unid de Urg de Teresina Prof Zenon Rocha Hut & Picos & 2 & $0,03 \%$ \\
SIH & Teresina & 4.194 & $73,84 \%$ \\
\hline
\end{tabular}

Fonte: SIH (2018).

O Hospital de Urgência de Teresina, é referência no Nordeste para tratamento de alta complexidade em traumatologia e ortopedia de urgência e emergência, conta vinte e quatro especialidades. Conforme os dados do próprio hospital apenas $36 \%$ dos pacientes atendidos residem em Teresina, os demais são provenientes do interior do estado e de outros estados como Maranhão, Pará e Tocantins. Dados que explicam os achados desta pesquisa visto que, a maioria dos traumatizados são atendidos neste estabelecimento, em virtude de ser um hospital de referência tanto do estado como de todo o Nordeste (TERESINA, 2018). Vale ressaltar que em alguns municípios do Estado, também fazem atendimento e hospitalização de pacientes vítimas de acidentes e traumas, o que observou - se como registro o hospital do município de Floriano com 10,40\% (591) ocorrências, seguido do município de Campo Maior 3,05\% (173) ocorrências. Destaca-se ainda que os pacientes que necessitam de um tratamento de alta complexidade são esses que migram para a referência de Teresina. 
A Tabela 5 mostra a distribuição das vítimas de acidentes de moto segundo o mês de ocorrência de internação, onde foi evidenciado que o mês de janeiro teve um contingente maior de ocorrências com cerca de $9,26 \%$ ( $n=526)$ e, apresentando o menor percentual o mês de dezembro, com um total de 4,05\% ( $n=230)$.

Tabela 5 - Caracterização das vítimas de acidentes motociclísticos atendidas no estado do Piauí segundo o mês de ocorrência de internação.

\begin{tabular}{lll}
\hline Variável & $\mathbf{N}$ & $\%$ \\
\hline Mês do atendimento & & \\
Janeiro & 526 & $9,26 \%$ \\
Fevereiro & 460 & $8,10 \%$ \\
Março & 498 & $8,77 \%$ \\
Abril & 520 & $9,15 \%$ \\
Maio & 485 & $8,54 \%$ \\
Junho & 389 & $6,85 \%$ \\
Julho & 495 & $8,71 \%$ \\
Agosto & 496 & $8,73 \%$ \\
Setembro & 491 & $8,64 \%$ \\
Outubro & 460 & $8,10 \%$ \\
Novembro & 437 & $7,69 \%$ \\
Dezembro & 230 & $4,05 \%$ \\
\hline
\end{tabular}

Fonte: $\mathrm{SIH}(2018)$.

Segundo Costa GS et al. (2018), o mês de janeiro, foi o de maior número de acidentes, confirmando dados do Departamento Nacional de Trânsito (DENATRAN), que segundo divulgações do CIPTRAN (Companhia Independente de Policiamento de Transito), o mês de janeiro, representou um crescimento de 3,48 no número de ocorrências no ano de 2014 nas vias públicas de Teresina-PI, dados esses que vão de encontro com o presente estudo.

Observou-se uma queda crescente de agosto a dezembro e um aumento de junho a agosto, mas os maiores números correspondem ao início do ano. Acredita-se que esteja relacionado ao período de férias onde há um aumento do consumo de bebidas alcoólicas e drogas onde há maior imprudência, aumentando assim o número dos acidentes, que tem como consequência a hospitalização ou o óbito. Pode ser justificado também por ser um mês chuvoso ocasionando o deslizamento em pistas molhadas, buracos encobertos por águas de chuva e visibilidade reduzida.

\section{CONCLUSÃO}

Diante dos resultados apresentados, conclui-se que no estado do Piauí, no ano de 2017, a maioria dos acidentados eram do sexo masculino, com a predominância de idade de 20 a 39 anos da cor parda, onde o principal mecanismo causador não foi especificado, onde foi observado um grande número de registos não especificados, dificultando uma visão real dos acidentes, sendo necessário um trabalho mais efetivo nos hospitais para que as informações essenciais sejam dispostas nos registros hospitalares referentes aos acidentes, não voltado apenas para os acidentes de moto.

O hospital de referência para traumatologia no estado em Piauí foi o que mais recebeu vítimas de acidentes de moto com trauma, onde o principal mês de ocorrências foi janeiro, com uma média de dias de internação de quatro dias e meio. $O$ estudo demostrou os elevados índices de acidentes com motociclistas no estado do Piauí, demostrando a necessidade da implantação de políticas públicas voltadas para a conscientização e educação no trânsito, como também de um trabalho educativo junto a essa população de usuários de motocicletas, visto que dar conhecimento e visibilidade do comprimento das leis de transito trará grandes benefícios a comunidade, a população na redução dos danos à saúde. 


\section{REFERÊNCIAS}

1. ANDRADE ML. Acidentes de motocicleta: características das vítimas e dos acidentes em hospital de Fortaleza - CE, Brasil. Rev. Rene, 2009; 10(4):52-59.

2. ANDRADE SSCA, JORGE MHPM. Internações hospitalares por lesões decorrentes de acidente de transporte terrestre no Brasil, 2013: permanência e gastos. Epidemiol. Serv. Saúde, 2017; 26(1):31-38.

3. BRASIL. Ministério da Saúde. Política nacional de redução da morbimortalidade por acidentes e violências. Brasília: Ministério da Saúde, 2000.

4. BRASIL. Ministério da Saúde (MS): Acidentes de moto nos país. 2015. Disponível em: http://www.brasil.gov.br/saude/2015/05/saudediscute-acoes-para-diminuir Acesso em: 08 nov. 2018.

5. BRASIL. Ministério da Saúde. Secretaria de Vigilância em Saúde. Viva: vigilância de violências e acidentes: 2013 e 2014. Brasília: Ministério da Saúde, 2017.

6. CORGOZINHO MM et al. Vulnerabilidade sobre duas rodas: tendência e perfil demográfico da mortalidade decorrente da violência no trânsito motociclístico no Brasil, 2004-2014. Cad Saúde Colet, 2018; 26(1):92-99.

7. COSTA GS et al. Perfil do Motociclista Traumatizado Atendido em um Hospital do Município de Teresina - Pi. Revista Interdisciplinar, 2018; 11(3):36-45.

8. LIBERATTI CLB et al. Uso de capacete por vítimas de acidentes de motocicleta em Londrina, sul do Brasil. Rev Panam Salud Publica/Pan Am J Public Health, 2003;13(1).

9. MASCARENHAS MDM, BARROS MBA. Caracterização das internações hospitalares por causas externas no sistema público de saúde, Brasil, 2011. Rev Bras Epidemiol, 2015; 18(4):771-784.

10. MALTA DC et al. Lesões no trânsito e uso de equipamento de proteção na população brasileira, segundo estudo de base populacional. Ciência e Saúde Coletiva, 2016; 21(2):399- 410.

11. MELO LMF et al. Perfil epidemiológico dos motociclistas acidentados no município de Manhuaçu- Minas Gerais. I Seminário Científico da FACIG - 29, 30 e 31 de outubro de 2015.

12. OLIVEIRA NLB, SOUSA RMC. Factors associated with the death of motorcyclists in traffic acidente. Rev. Esc. Enferm. USP, 2012; 46(6):1380-7

13. ORGANIZAÇÃO MUNDIAL DE SAÚDE (OMS). Relatório global sobre o estado da segurança viária 2015. Genebra: Organização Mundial de Saúde; 2015.

14. SILVA PHNV et al. Spatial study of mortality in motorcycle accidents in the State of Pernambuco, Northeastern Brazil. Rev Saude Publica, 2011;45(2):1-6.

15. SILVEIRA JZM, SOUZA JC. Sequelas de acidentes de trânsito e impactos na qualidade de vida. Revista Saúde e Pesquisa, 2016; 9(2):373-380.

16. SIMONETI FS et al. Padrão de vítimas e lesões no trauma com motocicletas. Revista da Faculdade de Ciências Médicas de Sorocaba, 2016; 18(1):36-40.

17. SOARES LS et al. Caracterização das vítimas de traumas por acidente com motocicleta internadas em um hospital público. Rev enferm UERJ, 2015; 23(1):115-21.

18. RODRIGUES CL et al. Acidentes que envolvem motociclistas e ciclistas no município de São Paulo: caracterização e tendências. Rev Bras Ortop, 2014; 49(6):602-606.

19. TERESINA-PI. Fundação Municipal de Saúde (FMS). Hospital de Urgência de Teresina é referência em traumas e recebe pacientes até de outros países. Disponível em: http://www.portalpmt.teresina.pi.gov.br/noticia/HUT-e-referencia-em-traumas-e-recebe-pacientes -ate-de-outros-paises/18273. Acesso em: 22 nov. 2018. 